\title{
Anomaly constraint on chiral central charge of $(2+1) d$ topological order
}

\author{
Ryohei Kobayashi \\ Institute for Solid State Physics, University of Tokyo, Kashiwa, Chiba 277-8583, Japan
}

(Received 5 January 2021; revised 16 March 2021; accepted 12 April 2021; published 10 May 2021)

\begin{abstract}
In this short paper, we argue that the chiral central charge $c_{-}$of a $(2+1)$ d topological ordered state is sometimes strongly constrained by 't Hooft anomaly of antiunitary global symmetry. For example, if a $(2+1) \mathrm{d}$ fermionic topological quantum field theory (TQFT) has a time-reversal anomaly with $T^{2}=(-1)^{F}$ labeled as $v \in \mathbb{Z}_{16}$, the TQFT must have $c_{-}=1 / 4 \bmod 1 / 2$ for odd $v$, while $c_{-}=0 \bmod 1 / 2$ for even $v$. This generalizes the fact that the bosonic TQFT with $T$ anomaly in a particular class must carry $c_{-}=4 \bmod 8$ to fermionic cases. We also study such a constraint for fermionic TQFT with $U(1) \times C T$ symmetry, which is regarded as a gapped surface of the topological superconductor in class AIII.
\end{abstract}

DOI: 10.1103/PhysRevResearch.3.023107

\section{INTRODUCTION}

The 't Hooft anomaly in quantum field theory is a mild violation of the conservation law due to quantum effects. It is well known that the 't Hooft anomaly constrains the low-energy behavior of the system, since we need nontrivial degrees of freedom in IR to match the given anomaly. For example, the seminal Lieb-Schultz-Mattis theorem [1-3] and its generalizations [4-8] provide strong spectral constraints on quantum systems on a lattice, which are understood as the consequence of 't Hooft anomaly involving lattice spatial symmetries that behave internally in infrared [9-15].

The 't Hooft anomaly is typically matched by a symmetrybroken or gapless phase (e.g., perturbative anomaly), but in some cases the anomaly is known to be matched by a symmetry-preserving gapped phase, realized by topological quantum field theory (TQFT) enriched by the global symmetry [16-30]. This implies that an anomaly in some particular class can be carried by topological degrees of freedom, not by gapless particles, and in particular, the system with an anomaly can have an energy gap. Recently it was also discovered that some global anomalies cannot be matched by a symmetry-preserving TQFT and lead to even stronger spectral constraints [31-33].

In this paper we work on symmetry-preserving TQFT with 't Hooft anomaly in $(2+1)$ dimensions and explore the constraints on the gapped phase enforced by the anomaly. We find that the chiral central charge $c_{-}$of the TQFT is strongly constrained by the 't Hooft anomaly of antiunitary global symmetry. This can be understood as a constraint on thermal Hall conductance observed on the surface state of a topological superconductor based on time-reversal symmetry. The result

Published by the American Physical Society under the terms of the Creative Commons Attribution 4.0 International license. Further distribution of this work must maintain attribution to the author(s) and the published article's title, journal citation, and DOI. of this paper also implies that the $(2+1)$ d topological ordered state with an anomalous $T$ symmetry of a specific index must have a quantized energy current on the $(1+1) \mathrm{d}$ boundary, which is proportional to the chiral central charge [34].

Let us illustrate what we mean by the chiral central charge of a $(2+1) d$ gapped phase. If there is the boundary for the $(2+1) d$ gapped phase realized by a $(1+1)$ d Conformal Field Theory (CFT), we can define the chiral central charge $c_{-}$via the chiral central charge of $(1+1)$ d CFT on the boundary. As a canonical example, the gravitational Chern-Simons theory $\mathrm{CS}_{\text {grav }}$ has $c_{-}=1 / 2$ on the boundary. We can also observe the chiral nature of $(2+1)$ d gapped theory as a sort of quantum anomaly of the $(2+1) d$ gapped phase, even without making up a $(1+1)$ d boundary. Namely, the gravitational Chern-Simons term has the framing anomaly characterized by the bulk topological action $\operatorname{Tr}(R \wedge R) /(192 \pi)$, since the gravitational Chern-Simons theory is defined on a spin manifold as

$$
\int_{M=\partial W} \mathrm{CS}_{\text {grav }}=\pi \int_{W} \widehat{A}(R)=\frac{1}{192 \pi} \int_{W} \operatorname{Tr}(R \wedge R) .
$$

Once we know the anomaly $\operatorname{Tr}(R \wedge R) /(192 \pi)$ can be expressed as the gravitational Chern-Simons theory, and once we know $\mathrm{CS}_{\text {grav }}$ has $c_{-}=1 / 2$, then we can combine them together to find that $\operatorname{Tr}(R \wedge R) /(192 \pi)$ implies $c_{-}=1 / 2$. Thus we can say that the $(2+1) \mathrm{d}$ TQFT has the chiral central $c_{-}$, if the theory has the framing anomaly given by

$$
\frac{c_{-}}{96 \pi} \operatorname{Tr}(R \wedge R) \text {. }
$$

Now we summarize the result of this paper. We start with time-reversal symmetry with $T^{2}=(-1)^{F}$ of fermionic TQFT (known as class DIII [35-37]), whose anomaly is classified by $\mathbb{Z}_{16}$ [17]. We show that if the TQFT has a $T$ anomaly labeled by an odd index $v \in \mathbb{Z}_{16}$, the TQFT must carry $c_{-}=1 / 4 \bmod$ $1 / 2$, while for even $v \in \mathbb{Z}_{16}$, the TQFT must instead carry $c_{-}=0 \bmod 1 / 2$.

We also consider $T$ anomaly in bosonic TQFT and show that we must have $c_{-}=4 \bmod 8$ for some particular class of the anomaly, while $c_{-}=0 \bmod 8$ for the other class. This 


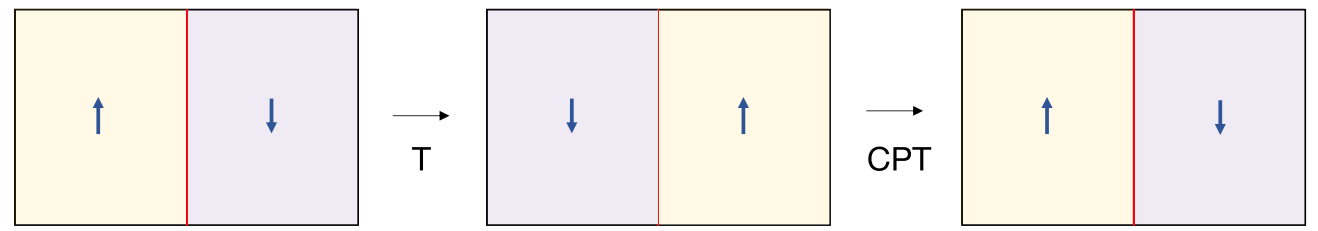

FIG. 1. The illustration for the $T$ domain wall. The $T$ domain wall separates the two distinct vacua in the $T$ broken phase. The $T$ symmetry acts on the figure by changing two vacua (i.e., yellow $\leftrightarrow$ purple). Since the $C P T$ commutes with $T$ (up to fermion parity), the $C P T$ leaves the vacua of the $T$-broken phase invariant and acts as the parity that reflects the figure across the domain wall. $T$ alone cannot be a symmetry on the domain wall since it flips the domain, but $T \cdot\left(C P_{\perp} T\right)$ works as the symmetry on the wall, since $C P_{\perp} T$ reflects back the configuration of domains.

result in the bosonic case is essentially known in [38], but we provide an alternative understanding for this phenomena, which is also applicable for fermionic cases. We also study a more involved fermionic TQFT with $U(1) \times C T$ symmetry (known as class AIII) and obtain a constraint $c_{-}=1 / 2 \bmod 1$ for a specific class of the anomaly regarded as a surface state of a topological superconductor [39].

\section{SPT PHASES ON TIME-REVERSAL SYMMETRY DEFECTS}

Let us consider a $(2+1) \mathrm{d}$ TQFT with the time-reversal symmetry $T$ that suffers from an 't Hooft anomaly. In our discussion, we couple the anomalous $(2+1) \mathrm{d}$ TQFT with a $(3+1) d$ Symmetry Protected Topological (SPT) phase based on the $T$ symmetry $^{1}$ and regard the anomalous $(2+1) \mathrm{d}$ TQFT as a boundary state of the $(3+1) \mathrm{d}$ SPT phase. In a general quantum theory with a global symmetry, there exists a codimension one topological operator that implements the symmetry action. We call this topological operator a symmetry defect. In the $(3+1) \mathrm{d} T$ SPT phase, let us consider a symmetry defect for the $T$ symmetry, which implements the orientation reversal of the space-time.

In general, for a $d$-dimensional SPT phase with $T$ symmetry, the $T$ symmetry defect itself becomes a SPT phase with the unitary $\mathbb{Z}_{2}$ symmetry. This phenomena can be understood in the phase where the $T$ symmetry is spontaneously broken. Then the $T$ defect is realized as a $T$ domain wall separating two distinct vacua of the symmetry-broken phase.

Concretely, let us consider an infinite system of the $(3+1) d T$ SPT phase and make up a $T$ domain wall of the

\footnotetext{
${ }^{1}$ In this paper we do not make a distinction between the SPT phases and the invertible field theories; what are referred to as SPT phases in the main text should more properly be called invertible phases. An invertible phase is a quantum field theory with a unique ground state on an arbitrary closed spatial manifold. An SPT phase is usually defined as an equivalence class of short-range-entangled gapped Hamiltonian systems with a specified symmetry. An SPT phase in this sense determines an equivalence class of invertible phases, by isolating its ground state, but it is a difficult and unsolved problem whether an arbitrary invertible phase associated to a global symmetry can be realized as an SPT phase in this sense. Invertible phases also include, e.g., the low-energy limit of the Kitaev wire, which is not counted as an SPT phase in the standard usage in the literature on condensed matter physics but is often called an SPT phase in the high-energy physics literature.
}

SPT phase by breaking the $T$ symmetry, dividing the system into the left and right domain. We are interested in a theory supported on the $T$ domain wall in this setup. To study the localized degrees of freedom on the domain wall, it is important to ask what the global symmetry of the domain wall is.

Throughout the paper we assume that the theory is Lorentz invariant. In that case we can find a global symmetry induced on the domain wall, with help of the $C P T$ symmetry [40,41]. Concretely, if the $T$ domain wall is located in a reflection symmetric fashion, the combined transformation of $T$ and $C P_{\perp} T$ fixes the domains, and thus acts solely on the domain wall. Here, $P_{\perp}$ denotes a spatial reflection fixing the configuration of the domain wall, see Fig. 1. Since the $C P T$ is antiunitary, the combined transformation $T \cdot\left(C P_{\perp} T\right)$ turns out to behave as a unitary $\mathbb{Z}_{2}$ symmetry on the domain wall. The theory on the $T$ domain wall is based on this induced $\mathbb{Z}_{2}$ symmetry.

In fact, there is a linear relation between the classification of the $(2+1) \mathrm{d}$ SPT phase on the $T$ symmetry defect and that of $(3+1) \mathrm{d} T$ SPT phases [40,41]. This relationship allows us to determine the classification of the $(3+1)$ d SPT phase from a given theory on the symmetry defect. This linear map between SPT classifications is nicely formulated in terms of the classification scheme of SPT phases given by cobordism group [42]. Here we briefly review the formulation of the map.

First, SPT phases in $(d+1)$ space-time dimension are classified by the cobordism group $\Omega_{\text {str }}^{d+1}$, where str stands for space-time structure that corresponds to the global symmetry, i.e., the choice of internal symmetry and the space-time symmetry such as fermion parity and/or time reversal [37,42-44]. If the structure group is the direct product of the internal symmetry $G$ and the space-time symmetry, we sometimes write the cobordism group in the form of $\Omega_{\text {space-time }}^{d+1}(B G)$, where space-time denotes the space-time symmetry.

Then, for a given $(d+1)$ d SPT phase with a structure group str and a codimension one symmetry defect of the $\mathbb{Z}_{2}$ global symmetry, we can define the linear map based on the induced structure on the symmetry defect,

$$
\Omega_{\mathrm{str}^{\prime}}^{d} \rightarrow \Omega_{\mathrm{str}}^{d+1},
$$

where $\operatorname{str}^{\prime}$ denotes the structure for the induced symmetry on the symmetry defect. This map of cobordism groups is called the Smith map.

For example, if we have unitary $\mathbb{Z}_{2}$ symmetry for the fermionic phase in a $(d+1)$ d space-time $X, X$ is equipped with spin structure on $T X$, and the $\mathbb{Z}_{2}$ gauge field. The SPT classification in $(d+1) \mathrm{d}$ is $\Omega_{\mathrm{str}}^{d+1}=\Omega_{\text {spin }}^{d+1}\left(B \mathbb{Z}_{2}\right)$. If we consider the $\mathbb{Z}_{2}$ symmetry defect $Y$ in $X$, the induced structure 
on $Y$ from that of $X$ is spin structure on $T Y \oplus N Y$, since $T X$ is decomposed into a tangent and normal bundle on $Y$. This structure is shown to be equivalent to $\operatorname{pin}^{-}$structure on $Y$. Thus we have the Smith map

$$
\Omega_{\text {pin }^{-}}^{d} \rightarrow \Omega_{\text {spin }}^{d+1}\left(B \mathbb{Z}_{2}\right),
$$

which reflects that antiunitary symmetry $T^{2}=1$ is induced on the symmetry defect from unitary $\mathbb{Z}_{2}$ via the $C P T$ theorem. The detailed description about the property of the Smith map is found in [40]. In the following discussions, we determine this linear Smith map by considering several cases that span the SPT classification we are interested in.

\section{A. $(3+1)$ d bosonic $T$ SPT phase}

In the bosonic case, the Smith map determines the classification of $(3+1) d T$ SPT phase from that of $(2+1) d \mathbb{Z}_{2}$ SPT phase on the $T$ symmetry defect, expressed as

$$
\Omega_{\mathrm{SO}}^{3}\left(B \mathbb{Z}_{2}\right) \rightarrow \Omega_{\mathrm{O}}^{4},
$$

where $\mathrm{SO}$ and $\mathrm{O}$ denote the oriented and unoriented structure, respectively. The SPT classification is $\Omega_{\text {SO }}^{3}\left(B \mathbb{Z}_{2}\right)=\mathbb{Z}_{2} \times \mathbb{Z}$, and $\Omega_{\mathrm{O}}^{4}=\mathbb{Z}_{2} \times \mathbb{Z}_{2}$. We label the elements of $\Omega_{\mathrm{SO}}^{3}\left(B \mathbb{Z}_{2}\right)$ as $\left(n_{D W}, n_{E}\right) \in \mathbb{Z}_{2} \times \mathbb{Z}$. The generators are described as follows:

(a) $(1,0)$ corresponds to the $\mathbb{Z}_{2}$ SPT phase given by the classical action

$$
\exp \left(\pi i \int a^{3}\right)
$$

with a $\mathbb{Z}_{2}$ gauge field $a$, which characterizes a nontrivial element of $H^{3}\left(B \mathbb{Z}_{2}, U(1)\right)=\mathbb{Z}_{2}$.

(b) $(0,1)$ corresponds to the $E_{8}$ state [45] with chiral central charge $c_{-}=8$.

Meanwhile, we label the $(3+1) \mathrm{d} T$ SPT classification by $\left(m_{1}, m_{2}\right) \in \mathbb{Z}_{2} \times \mathbb{Z}_{2}$, whose generators are described as follows:

(a) $(1,0)$ corresponds to the classical action

$$
\exp \left(\pi i \int w_{1}^{4}\right)
$$

where $\left[w_{1}\right] \in H^{1}\left(M, \mathbb{Z}_{2}\right)$ is the first Stiefel-Whitney class of the space-time $M$.

(b) $(0,1)$ corresponds to the classical action

$$
\exp \left(\pi i \int w_{2}^{2}\right)
$$

with $\left[w_{2}\right] \in H^{2}\left(M, \mathbb{Z}_{2}\right)$ as the second Stiefel-Whitney class.

The Smith map $\mathbb{Z}_{2} \times \mathbb{Z} \rightarrow \mathbb{Z}_{2} \times \mathbb{Z}_{2}$ for (2.3) is given in the form of

$$
\left(n_{D W}, n_{E}\right) \rightarrow\left(\alpha_{1} n_{D W}+\alpha_{2} n_{E}, \beta_{1} n_{D W}+\beta_{2} n_{E}\right),
$$

for coefficients $\alpha_{1}, \alpha_{2}, \beta_{1}, \beta_{2} \in \mathbb{Z}_{2}$. We determine these coefficients by finding what $(2+1) \mathrm{d}$ phases map to the action (2.5), (2.6), respectively. We will see that $\alpha_{1}=1, \alpha_{2}=$ $0, \beta_{1}=0, \beta_{2}=1$ in the following discussions.

We find the theory on the $T$ symmetry defect for (2.5) by twisted compactification of (2.5) with respect to the $T$ symmetry. It turns out that the restriction of the $T$ defect on the $T$ symmetry defect is regarded as the $\mathbb{Z}_{2}$ gauge field $a$, and the compactified action is given by (2.4). This determines $\alpha_{1}=1, \alpha_{2}=0$.

To find the theory on the $T$ symmetry defect for (2.6), it is convenient to consider the $(2+1) \mathrm{d}$ gapped boundary of the SPT phase that preserves the $T$ symmetry. The gapped boundary is realized by the $\mathbb{Z}_{2}$ gauge theory given by the action

$$
\exp \left(\pi i \int a \cup \delta b+a \cup w_{2}+b \cup w_{2}\right),
$$

with dynamical $\mathbb{Z}_{2}$ gauge fields $a, b$. This action realizes a TQFT known as the 3-fermion state [38,46,47], a $(2+1) \mathrm{d} \mathbb{Z}_{2}$ gauge theory whose electric and magnetic particle are both fermions. In general, a $(2+1) \mathrm{d}$ bosonic topological ordered state is described by the fusion and braiding properties of anyons, which are characterized by an algebraic theory of anyons known as a unitary modular tensor category (UTMC). For a given UTMC that describes a $(2+1) \mathrm{d}$ TQFT, there is a way to compute the chiral central charge $c_{-}$modulo 8 known as the Gauss-Milgram formula, given by $e^{2 \pi i c_{-} / 8}=$ $\sum_{a} d_{a}^{2} \theta_{a} / \mathcal{D}[45] .^{2}$

Here the sum is over anyons of the UTMC, $d_{a}$ is the quantum dimension, $\theta$ is the topological spin, and $\mathcal{D}$ is the total dimension given by $\mathcal{D}^{2}=\sum_{a} d_{a}^{2}$. According to this formula, we can immediately see that the 3 -fermion state has the chiral central charge $c_{-}=4 \bmod 8$.

Then, let us break the $T$ symmetry simultaneously in the $(3+1) \mathrm{d}$ bulk and the $(2+1) \mathrm{d}$ boundary, such that the $T$ domain wall in the bulk terminates on a domain wall at the boundary. The $T$ domain wall separates the left and right domain, see Fig. 2. Let us assume that one specific realization of our system has $c_{-}=4+8 m$ for $m \in \mathbb{Z}$, on the boundary of the left domain.

Since the right domain can be prepared as a partner of the left domain conjugate under the $T$ symmetry, the boundary of right domain carries $c_{-}=-(4+8 m)$, since the orientation at the right domain gets reversed by the $T$ action. This implies the $(3+1) \mathrm{d}$ SPT action given by

$$
\frac{c_{-}}{96 \pi} \operatorname{Tr}(R \wedge R)
$$

\footnotetext{
${ }^{2}$ We can see the correspondence of the Gauss-Milgram formula with the framing anomaly based on the following argument. Starting from the UTMC $\mathcal{C}$, we can construct the $(3+1) \mathrm{d}$ Walker-Wang model, a $(3+1)$ d SPT phase whose boundary is given by a $(2+1) \mathrm{d}$ TQFT described by the UTMC $\mathcal{C}$. Then, it has been shown in [38] that the partition function of the Walker-Wang TQFT on the complex projective space $\mathbb{C} \mathbb{P}^{2}$ produces the Gauss-Milgram formula

$$
Z\left(\mathbb{C P}^{2}\right)=\frac{1}{\mathcal{D}} \sum_{a} d_{a}^{2} \theta_{a} .
$$

Meanwhile, we can see that

$$
\exp \left(\int_{\mathbb{C P}^{2}} \frac{i c_{-}}{96 \pi} \operatorname{Tr}(R \wedge R)\right)=e^{2 \pi i c_{-} / 8},
$$

by recalling that $\mathbb{C} \mathbb{P}^{2}$ has the signature 1 . Hence, supposing that the Walker-Wang model is effectively described by the $R \wedge R$ action, the Gauss-Milgram formula exactly computes the framing anomaly $c_{-}$.
} 


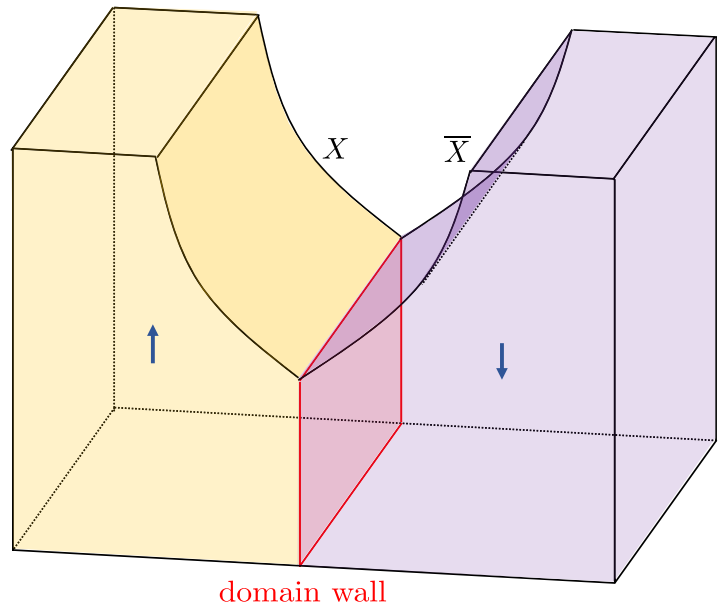

FIG. 2. The illustration for our setup. We have a $T$ domain wall (red plane) in the $(3+1) \mathrm{d}$ bulk, which ends at $\partial X$ on the boundary.

has a kink of $c_{-}$from $c_{-}=4+8 m$ to $c_{-}=-(4+8 m)$ on the domain wall; thus we obtain the gravitational Chern-Simons theory $(8+16 m) \cdot 2 \mathrm{CS}_{\text {grav }}$ on the $T$ domain wall, which carries $c_{-}=8 \bmod 16$. We note that the bulk action (2.11) with $c_{-}=4 \bmod 8$ gives the same action as (2.6) on a closed oriented manifold, since $w_{2}^{2}$ is related to the Pontryagin class as $[46,48]$

$$
w_{2}^{2}=p_{1} \quad \bmod 2,
$$

and then we have

$$
\exp \left(\pi i \int w_{2}^{2}\right)=\exp \left(\frac{i}{24 \pi} \int \operatorname{Tr}(R \wedge R)\right) .
$$

We can also understand the chiral domain wall with $c_{-}=8$ mod 16 as follows. We denote the $(2+1) \mathrm{d}$ space-time for the left domain on the gapped boundary as $X$, see Fig. 2. The $T$ domain wall in the bulk ends on $\partial X$ at the $(2+1) \mathrm{d}$ boundary. Since the $T$ symmetry is preserved on the boundary, $\partial X$ must support a $T$ defect operator of the $(2+1) \mathrm{d}$ TQFT on the boundary. Because the boundary is a gapped TQFT, the $T$ defect on $\partial X$ must be topological and carry gapped degrees of freedom, which must lead to $c_{-}=0$ on $\partial X$.

Now, the left and right domain of the TQFT on the boundary contributes $c_{-}=8 \bmod 16$ to $\partial X$, and it must be canceled by the bulk contribution. Thus the $T$ domain wall in the $(3+1) d$ SPT phase must carry $c_{-}=8 \bmod 16$. We identify the $c_{-}=8$ phase on the $T$ domain wall as the $E_{8}$ state that generates the free part of $\Omega_{\mathrm{SO}}^{3}\left(B \mathbb{Z}_{2}\right)=\mathbb{Z}_{2} \times \mathbb{Z}$. Thus we conclude that $\beta_{2}=1$ in the Smith map. We can further see that $\beta_{1}=0$ by seeing that the action given by decorating the $(2+1)$ d action $(2.4)$ on the $T$ domain wall evaluates $Z\left(\mathbb{C P}^{2}\right)=1$ since $\mathbb{C} \mathbb{P}^{2}$ is oriented and so cannot generate the action (2.6).

Summarizing, the Smith map (2.3) is given by

$$
\left(n_{D W}, n_{E}\right) \rightarrow\left(n_{D W}, n_{E}\right) \quad \bmod 2 .
$$

\section{B. $(3+1)$ d fermionic $T$ SPT phase: $T^{2}=(-1)^{F}$}

In the fermionic case, the $T$ symmetry $T^{2}=(-1)^{F}$ corresponds to $\mathrm{pin}^{+}$structure of the space-time. The Smith map determines the classification of $(3+1) \mathrm{d} T$ SPT phase from that of $(2+1) \mathrm{d} \mathbb{Z}_{2}$ SPT phase on the $T$ domain wall, expressed as

$$
\Omega_{\text {spin }}^{3}\left(B \mathbb{Z}_{2}\right) \rightarrow \Omega_{\text {pin }^{+}}^{4} .
$$

This gives a linear map $\mathbb{Z}_{8} \times \mathbb{Z} \rightarrow \mathbb{Z}_{16}$, where the $\mathbb{Z}$ part of $\Omega_{\text {spin }}^{3}\left(B \mathbb{Z}_{2}\right)$ is generated by the $p+i p$ superconductor with $c_{-}=1 / 2$. The $\mathbb{Z}_{8}$ part corresponds to the $\mathbb{Z}_{2}$ SPT phase described by the decoration of the Kitaev wire [49]. If we label elements as $(n, k) \in \mathbb{Z}_{8} \times \mathbb{Z}$ and $v \in \mathbb{Z}_{16}$, the map is determined by [40] in the form of

$$
v=2 n-k \quad \bmod 16 .
$$

In particular, the above formula dictates that the odd $v$ must carry odd $k$ on the $T$ domain wall. Namely, $c_{-}$of the SPT phase on the $T$ domain wall must be $c_{-}=1 / 2 \bmod 1$ when $v$ is odd and $c_{-}=0 \bmod 1$ when $v$ is even.

\section{CONSTRAINT ON $(2+1) \mathrm{d} \mathrm{pin}^{+}$AND BOSONIC TQFT}

We argue that the TQFT on the boundary of $(2+1) \mathrm{d}$ TQFT has a restricted value of $c_{-}$, depending on the chiral phase on the $T$ domain wall controlled by the Smith map. For simplicity, we focus on $\operatorname{pin}^{+}$anomaly classified by $\mathbb{Z}_{16}$. The generalization to the bosonic case is straightforward.

Let us consider a $(2+1) \mathrm{d} \mathrm{pin}^{+}$TQFT $\mathcal{T}$ on the boundary of a $(3+1) \mathrm{d} T$ SPT phase, classified by $v \in \mathbb{Z}_{16}$. We again work on the geometry described in Fig. 2, i.e., we break the $T$ symmetry simultaneously in the $(3+1) \mathrm{d}$ bulk and the $(2+1) \mathrm{d}$ boundary such that the $T$ domain wall in the bulk terminates on a domain wall at the boundary. If the boundary TQFT on the left domain $X$ has the chiral central charge $c_{-}=c_{\mathcal{T}}+m / 2$ for $m \in \mathbb{Z}$, the right domain $\bar{X}$ has $c_{-}=-\left(c_{\mathcal{T}}+m / 2\right)$, since it is the $T$ partner of the left domain. This implies the $(3+1)$ d SPT action given by

$$
\frac{c_{-}}{96 \pi} \operatorname{Tr}(R \wedge R)
$$

has a kink of $c_{-}$from $c_{-}=c_{\mathcal{T}}+m / 2$ to $c_{-}=-c_{\mathcal{T}}+m / 2$ on the $T$ domain wall; thus we obtain the gravitational ChernSimons theory $\left(2 c_{\mathcal{T}}+m\right) \cdot 2 \mathrm{CS}_{\text {grav }}$ on the $T$ domain wall, which carries $c_{-}=2 c_{\mathcal{T}}$ mod 1 . Meanwhile, according to the Smith map (2.16), $c_{-}$carried by the $T$ domain wall must be $c_{-}=v / 2 \bmod 1$. This leads to $c_{\mathcal{T}}=v / 4 \bmod 1 / 2$.

We can also understand the chiral domain wall with $c_{-}=$ $1 / 4 \bmod 1 / 2$ as follows. The $T$ domain wall in the bulk ends on $\partial X$ at the $(2+1) d$ boundary. Since the $T$ symmetry is preserved on the boundary, $\partial X$ must support a $T$ defect operator of the $(2+1) \mathrm{d}$ TQFT on the boundary. Because the boundary is a gapped TQFT, the $T$ defect on $\partial X$ must be topological and carry gapped degrees of freedom, which must lead to $c_{-}=0$ on $\partial X$. Due to the Smith map (2.16), the $T$ defect from the bulk contributes as $c_{-}=1 / 2 \bmod 1$ to $\partial X$, when $v$ is odd in $\mathbb{Z}_{16}$. Meanwhile, the TQFT on the boundary contributes $c_{-}=\left(c_{\mathcal{T}}+m / 2\right)+\left(c_{\mathcal{T}}+m / 2\right)=2 c_{\mathcal{T}} \bmod 1$.

Thus, in order to have $c_{-}=0$ on $\partial X$ for odd $v \in \mathbb{Z}_{16}$, we must have $2 c_{\mathcal{T}}=1 / 2 \bmod 1$, so $c_{\mathcal{T}}=1 / 4 \bmod 1 / 2$. For even $v \in \mathbb{Z}_{16}$, the $T$ defect instead carries $c_{-}=0 \bmod 1$, so we have $c_{\mathcal{T}}=0 \bmod 1 / 2$.

For the bosonic case, a similar argument shows that $c_{-}=4$ mod 8 if the $(2+1)$ d TQFT has an anomaly characterized by the SPT action $\int w_{2}^{2}$, otherwise $c_{-}=0 \bmod 8$. This is 
also understood by the fact that $c_{-} \bmod 8$ is diagnosed by the partition function of the bulk $(3+1) d$ SPT phase on $\mathbb{C} \mathbb{P}^{2}$ that corresponds to the Gauss-Milgram formula [38], $Z_{\mathrm{SPT}}\left(\mathbb{C P}^{2}\right)=e^{2 \pi i c_{-} / 8}$, which is -1 for $\int w_{2}^{2}$ and 1 for $\int w_{1}^{4}$.

\section{IV. $(3+1)$ d TOPOLOGICAL SUPERCONDUCTOR IN CLASS AIII}

Here, let us apply our argument to the $(3+1) \mathrm{d}$ SPT phase with $U(1) \times C T$ symmetry (called class AIII). This structure corresponds to the structure group $\operatorname{pin}^{c}:=\left(\operatorname{pin}^{ \pm} \times\right.$ $U(1)) / \mathbb{Z}_{2}$, where $C T$ corresponds to the orientation reversing element of $\operatorname{pin}^{ \pm}$which commutes with $U(1)$.

Let us consider the $C T$ defect of the $\operatorname{pin}^{c}(3+1) \mathrm{d}$ SPT phase. To see the induced structure on the $C T$ domain wall, it is convenient to regard $\operatorname{pin}^{c}$ as a pin ${ }^{+}$structure twisted by $U(1)$. pin $^{+}$induces oriented spin structure equipped with the $\mathbb{Z}_{2}$ symmetry on the domain wall, and we also have $U(1)$ symmetry that twists the induced spin structure. Then, the induced structure on the domain wall becomes $\operatorname{spin}^{c}$ with $\mathbb{Z}_{2}$ symmetry.

Therefore we have the Smith map for cobordism groups

$$
\Omega_{\operatorname{spin}^{c}}^{3}\left(B \mathbb{Z}_{2}\right) \rightarrow \Omega_{\text {pin }^{c}}^{4} .
$$

The bordism or cobordism groups for these structures are studied in [50-52] and given by $\Omega_{\operatorname{spin}^{c}}^{3}\left(B \mathbb{Z}_{2}\right)=\mathbb{Z}_{4} \times \mathbb{Z} \times \mathbb{Z}$, and $\Omega_{\text {pin }^{c}}^{4}=\mathbb{Z}_{8} \times \mathbb{Z}_{2}$. We label the elements of $\Omega_{\text {spin }^{c}}^{3}\left(B \mathbb{Z}_{2}\right)$ as $\left(n_{4}, n_{C S}, n_{E}\right) \in \mathbb{Z}_{4} \times \mathbb{Z} \times \mathbb{Z}$. The generators are described as follows:

(a) $(0,1,0)$ corresponds to the $\operatorname{spin}^{c}$ Chern-Simons theory at level 1 , defined via the $(3+1) \mathrm{d} \operatorname{spin}^{c} \theta$-term in (4.5). This theory carries $c_{-}=1$.

(b) $(0,0,1)$ corresponds to the $E_{8}$ state, which carries $c_{-}=$

(c) $(1,0,0)$ generates the $\mathbb{Z}_{4}$ group, and we believe that it should be formulated in a similar way to the Gu-Wen $\mathbb{Z}_{2}$ SPT phase based on spin structure, which is labeled by a pair of cohomological data [53,54]. Actually, if we compute the cobordism group by using the toolkit of the AtiyahHilzebruch spectral sequence (AHSS) [51], we see that it can also be described by a pair of cohomological data, which should be regarded as the $\operatorname{spin}^{c}$ version of the $\mathrm{Gu}$-Wen phase. Namely, the group $\mathbb{Z}_{4}$ is the nontrivial extension of

$$
H^{2}\left(B \mathbb{Z}_{2}, \Omega_{\text {spin }^{c}}^{1}\right)=H^{2}\left(B \mathbb{Z}_{2}, \mathbb{Z}\right)=\mathbb{Z}_{2}
$$

by

$$
H^{4}\left(B \mathbb{Z}_{2}, \Omega_{\operatorname{spin}^{c}}^{-1}\right)=H^{3}\left(B \mathbb{Z}_{2}, U(1)\right)=\mathbb{Z}_{2} .
$$

So we expect that the $\mathbb{Z}_{2}$ subgroup of the $\mathbb{Z}_{4}$ is given by the bosonic $\mathbb{Z}_{2}$ SPT phase with the classical action

$$
\exp \left(\pi i \int a^{3}\right),
$$

which characterizes the nontrivial element of $H^{3}\left(B \mathbb{Z}_{2}, U(1)\right)=\mathbb{Z}_{2}$. Based on the analogy with the Gu-Wen spin SPT phase, we believe that $H^{2}\left(B \mathbb{Z}_{2}, \Omega_{\text {spin }^{c}}^{1}\right)$ is associated with the physical description that a $(0+1) \mathrm{d} \operatorname{spin}^{c} \mathrm{SPT}$ phase (in this case a complex fermion with charge 1) is decorated on the junction of $\mathbb{Z}_{2}$ defects, and the way for the decoration is controlled by $H^{2}\left(B \mathbb{Z}_{2}, \Omega_{\text {spin }^{c}}^{1}\right)$. Though we have not constructed any action for the $\mathbb{Z}_{4}$ generator, we believe that there exists a good state sum definition for this theory on lattice like the Gu-Wen spin SPT phases, which carries $c_{-}=0$.

Meanwhile, if we label the element of $\Omega_{\operatorname{pin}^{c}}^{4}$ as $\left(m_{8}, m_{2}\right) \in$ $\mathbb{Z}_{8} \times \mathbb{Z}_{2}$, the actions for the generators are described as follows:

(a) If the space-time is oriented, the generator $\mathbb{Z}_{8},(1,0)$ is described by the $\theta$-term for $\operatorname{spin}^{c}$ gauge field at $\theta=\pi[55,56]$, given by

$$
S[a]=i \theta\left(\frac{1}{2(2 \pi)^{2}} \int f \wedge f-\frac{\sigma}{8}\right),
$$

where $a$ is a $\operatorname{spin}^{c}$ gauge field with the Dirac quantization condition

$$
\int_{C} \frac{f}{2 \pi}=\frac{1}{2} \int_{C} w_{2} \quad \bmod 1
$$

for any oriented $2 \mathrm{~d}$ cycle in the space-time. $\sigma$ denotes the signature of the manifold. Also, for later convenience, we note that $m_{8}=4 \in \mathbb{Z}_{8},(4,0)$ is given by

$$
\exp \left(\pi i \int w_{1}^{4}\right) \text {. }
$$

(b) The generator of $\mathbb{Z}_{2},(0,1)$ is given by

$$
\exp \left(\pi i \int w_{2}^{2}\right) \text {. }
$$

Then we can almost completely determine the Smith map (4.1) by considering a $C T$ domain wall of the $(3+1) d$ action. First, since we know in Sec. II A that the $(3+1)$ d action $(4.7)$ localizes the action (4.4) on the domain wall, we expect that $(2,0,0)$ is mapped to $(4,0)$ by the Smith map. Due to linearity of the Smith map, it shows that $\mathbb{Z}_{4}$ part of $\Omega_{\text {spin }^{c}}^{3}\left(B \mathbb{Z}_{2}\right)$ is mapped to the $\mathbb{Z}_{4}$ subgroup of the $\mathbb{Z}_{8}$ part in $\Omega_{\text {pin }}^{4}$. According to Sec. II A, we also know that the $(3+1) \mathrm{d}$ action $(4.8)$ for $(0,1)$ localizes the $E_{8}$ state on the domain wall, so it also determines how $(0,0,1)$ transforms. Finally, for $(1,0)$ in $\Omega_{\text {pin }}^{4}$, the $C T$ domain wall for the $(3+1) \mathrm{d} \theta$-term induces a kink of $\theta$ from $\theta=+\pi$ to $\theta=-\pi$, so we obtain a Chern-Simons theory at level 1 on the domain wall. So we know how $(0,1,0)$ transforms. Thus our Smith map is obtained as

$$
\left(n_{4}, n_{C S}, n_{E}\right) \rightarrow\left[n_{C S}+(2+4 p) n_{4}, n_{E}\right],
$$

where $p$ is an undetermined integer.

According to the Smith map, the odd element of $\mathbb{Z}_{8}$ in $\Omega_{\text {pin }}^{4}$ must carry $c_{-}=1 \bmod 2$ on the $C T$ domain wall. So by using the same logic as the pin $^{+}$anomaly, we can see that the odd phase in $\mathbb{Z}_{8}$ must have $c_{-}=1 / 2 \bmod 1$.

\section{DISCUSSION}

In this paper we found the anomaly constraint on chiral central charge of $(2+1)$ d topological order with $T$ symmetry. The constraint comes from a chiral state localized on the $T$ domain wall in the bulk SPT phase. It should be interesting to study such a constraint on the $(2+1) \mathrm{d}$ TQFT enriched by more generic global symmetry, though we have only studied the cases for $T$ and $U(1) \times C T$. For example, by using the AHSS, $(d+1)$ d fermionic SPT phases with $G_{b}$ symmetry are 
generally labeled by the set of cohomological data [57,58],

$$
n_{p} \in H^{p}\left(B G_{b}, \Omega_{\mathrm{spin}}^{d+1-p}\right),
$$

for $0 \leqslant p \leqslant d+2$. Here, $G_{b}$ can contain time reversal, and the full global symmetry is described by $G_{f}$, defined as the symmetry extension by fermion parity $\mathbb{Z}_{2}^{F} \rightarrow G_{f} \rightarrow G_{b}$. The data $n_{p}$ is associated with the description of the SPT phase based on the decorated domain wall; $n_{p}$ controls the way to decorate an $[(d-p)+1]$ d SPT phase on the domain wall of $G_{b}$. Specifically, the nontrivial $n_{1}$ implies the decoration of $p+i p$ superconductor on the $G_{b}$ domain wall. We expect that this description of decorated domain wall leads to a unified formulation of the anomaly constraints on the Hilbert space for a broad class of global symmetries. See also Ref. [59].

It is also very interesting to study the constraint on the chiral central charge for crystalline symmetries. In that case there is a simple way to reduce the $(3+1)$ d SPT phase to the lower-dimensional system with internal symmetries. For example, consider a unitary reflection symmetry across the $(2+1) d$ plane which protects the $(3+1) d$ SPT phase. Then we can operate the unitary circuit respecting the reflection symmetry away from the reflection plane, which can disentangle the SPT phase away from the reflection plane. After all, we obtain the reduced $(2+1)$ d SPT phase supported on the reflection plane, where the reflection symmetry acts internally. Based on this logic, [60] obtains a constraint on chiral central charge for the $(3+1)$ d SPT phase with the spatial reflection symmetry.

In the present paper, we worked on the setup with Lorentz invariance and did not discuss the effect of lattice. It is interesting what the Smith map and the global symmetry on the symmetry defects looks like in lattice systems, since we deal with lattice systems in the study of SPT phases which are not Lorentz invariant in general. There is a lattice model for the (3+1)d SPT phase with $T^{2}=(-1)^{F}$ [61], where in the $T$ broken phase we observe a unitary global $\mathbb{Z}_{2}$ symmetry on the domain wall, at the level of a lattice model. We believe that this global symmetry reflects the induced $\mathbb{Z}_{2}$ symmetry via the CPT theorem of the effective field theory. It is interesting to study such a lattice model in more detail, e.g., the gapped boundary of this theory, and see how the anomaly constraint works.

Finally, another interesting direction is to ask if our constraint on $c_{-}$is applicable to generic gapless phases, while we have worked on gapped topological ordered states in the present paper. We leave these problems to future work.

\section{ACKNOWLEDGMENTS}

The author is grateful to Maissam Barkeshli and Kantaro Ohmori for enlightening discussions. The author also thanks Yunqin Zheng for helpful comments on the manuscript. The author is supported by the Japan Society for the Promotion of Science (JSPS) through Grant No. 19J20801.

scopic Constraints on Symmetry-Enriched Topological Phases: A View from the Surface, Phys. Rev. X 6, 041068 (2016).

[12] M. A. Metlitski and R. Thorngren, Intrinsic and emergent anomalies at deconfined critical points, Phys. Rev. B 98, 085140 (2018).

[13] Y. Yao, C.-T. Hsieh, and M. Oshikawa, Anomaly Matching and Symmetry-Protected Critical Phases in SU(n) Spin Systems in 1+1 Dimensions, Phys. Rev. Lett. 123, 180201 (2019).

[14] T. Sulejmanpasic and Y. Tanizaki, C-P-T anomaly matching in bosonic quantum field theory and spin chains, Phys. Rev. B 97, 144201 (2018).

[15] Y. Tanizaki and T. Sulejmanpasic, Anomaly and global inconsistency matching: $\theta$-angles, $S U(3) / U(1)^{2}$ nonlinear sigma model, $S U$ (3) chains and its generalizations, Phys. Rev. B 98, 115126 (2018).

[16] F. J. Burnell, X. Chen, L. Fidkowski, and A. Vishwanath, Exactly soluble model of a 3D symmetry protected topological phase of bosons with surface topological order, Phys. Rev. B 90, 245122 (2014)

[17] L. Fidkowski, X. Chen, and A. Vishwanath, Non-Abelian Topological Order on the Surface of a 3d Topological Superconductor from an Exactly Solved Model, Phys. Rev. X 3, 041016 (2013).

[18] C. Wang, A. C. Potter, and T. Senthil, Gapped symmetry preserving surface-state for the electron topological insulator, Phys. Rev. B 88, 115137 (2013).

[19] P. Bonderson, C. Nayak, and X.-1. Qi, A time-reversal invariant topological phase at the surface of a 3D topological insulator, J. Stat. Mech. (2013) P09016. 
[20] X. Chen, L. Fidkowski, and A. Vishwanath, Symmetry enforced non-Abelian topological order at the surface of a topological insulator, Phys. Rev. B 89, 165132 (2014).

[21] M. A. Metlitski, C. Kane, and M. Fisher, A symmetryrespecting topologically-ordered surface phase of $3 \mathrm{~d}$ electron topological insulators, Phys. Rev. B 92, 125111 (2015).

[22] X. Chen, F. J. Burnell, A. Vishwanath, and L. Fidkowski, Anomalous Symmetry Fractionalization and Surface Topological Order, Phys. Rev. X 5, 041013 (2015).

[23] A. Kapustin and R. Thorngren, Anomalous Discrete Symmetries in Three Dimensions and Group Cohomology, Phys. Rev. Lett. 112, 231602 (2014).

[24] M. A. Metlitski, L. Fidkowski, X. Chen, and A. Vishwanath, Interaction effects on 3D topological superconductors: Surface topological order from vortex condensation, the 16 fold way and fermionic Kramers doublets, arXiv:1406.3032.

[25] R. Thorngren and C. von Keyserlingk, Higher SPT's and a generalization of anomaly in-flow, arXiv:1511.02929.

[26] E. Witten, The "parity" anomaly on an unorientable manifold, Phys. Rev. B 94, 195150 (2016).

[27] J. Wang, X.-G. Wen, and E. Witten, Symmetric Gapped Interfaces of SPT and SET States: Systematic Constructions, Phys. Rev. X 8, 031048 (2018).

[28] R. Kobayashi, K. Ohmori, and Y. Tachikawa, On gapped boundaries for SPT phases beyond group cohomology, J. High Energy Phys. 11 (2019) 131.

[29] R. Kobayashi, Pin TQFT and Grassmann integral, J. High Energy Phys. 12 (2019) 014.

[30] D. Bulmash and M. Barkeshli, Absolute anomalies in $(2+1) \mathrm{d}$ symmetry-enriched topological states and exact $(3+1) \mathrm{d}$ constructions, Phys. Rev. Research 2, 043033 (2020).

[31] C. Córdova and K. Ohmori, Anomaly obstructions to symmetry preserving gapped phases, arXiv:1910.04962.

[32] C. Córdova and K. Ohmori, Anomaly constraints on gapped phases with discrete chiral symmetry, Phys. Rev. D 102, 025011 (2020).

[33] R. Thorngren, TQFT, symmetry breaking, and finite gauge theory in 3+1d, Phys. Rev. B 101, 245160 (2020).

[34] A. Kapustin and L. Spodyneiko, Thermal Hall conductance and a relative topological invariant of gapped two-dimensional systems, Phys. Rev. B 101, 045137 (2020).

[35] A. P. Schnyder, S. Ryu, A. Furusaki, and A. W. W. Ludwig, Classification of topological insulators and superconductors in three spatial dimensions, Phys. Rev. B 78, 195125 (2008).

[36] A. Kitaev, Periodic table for topological insulators and superconductors, in Advances in Theoretical Physics: Landau Memorial Conference, edited by V. Lebedev and M. Feigel'man, AIP Conf. Proc. No. 1134 (American Institute of Physics (AIP), College Park, Maryland, 2009), p. 22.

[37] D. S. Freed and M. J. Hopkins, Reflection positivity and invertible topological phases, arXiv:1604.06527.

[38] M. Barkeshli, P. Bonderson, C.-M. Jian, M. Cheng, and K. Walker, Reflection and time reversal symmetry enriched topological phases of matter: Path integrals, non-orientable manifolds, and anomalies, Commun. Math. Phys. 374, 1021 (2020).

[39] C. Wang and T. Senthil, Interacting fermionic topological insulators/superconductors in three dimensions, Phys. Rev. B 89, 195124 (2014).

[40] I. Hason, Z. Komargodski, and R. Thorngren, Anomaly matching in the symmetry broken phase: Domain walls, CPT, and the Smith isomorphism, SciPost Phys. 8, 062 (2020).

[41] C. Córdova, K. Ohmori, S.-H. Shao, and F. Yan, Decorated $\mathbb{Z}_{2}$ symmetry defects and their time-reversal anomalies, Phys. Rev. D 102, 045019 (2020).

[42] A. Kapustin, R. Thorngren, A. Turzillo, and Z. Wang, Fermionic symmetry protected topological phases and cobordisms, J. High Energy Phys. 12 (2015) 1.

[43] A. Kapustin, Symmetry protected topological phases, anomalies, and cobordisms: Beyond group cohomology, arXiv:1403.1467.

[44] K. Yonekura, On the cobordism classification of symmetry protected topological phases, Commun. Math. Phys. 3681121 (2019).

[45] A. Kitaev, Anyons in an exactly solved model and beyond, Ann Phys. 321, 2 (2006).

[46] P.-S. Hsin and S.-H. Shao, Lorentz symmetry fractionalization and dualities in $(2+1)$ d, SciPost Phys. 8 (2020).

[47] R. Thorngren, Framed Wilson operators, fermionic strings, and gravitational anomaly in 4d, J. High Energy Phys. 02 (2015) 152.

[48] O. Aharony, N. Seiberg, and Y. Tachikawa, Reading between the lines of four-dimensional gauge theories, J. High Energy Phys. 08 (2013) 115.

[49] N. Tarantino and L. Fidkowski, Discrete spin structures and commuting projector models for $2 \mathrm{~d}$ fermionic symmetry protected topological phases, Phys. Rev. B 94, 115115 (2016).

[50] M. Guo, P. Putrov, and J. Wang, Time reversal, $S U(n)$ Yang-Mills and cobordisms: Interacting topological superconductors/insulators and quantum spin liquids in $3+1 \mathrm{~d}$, Ann. Phys. 394, 244 (2018).

[51] I. García-Etxebarria and M. Montero, Dai-Freed anomalies in particle physics, J. High Energy Phys. 08 (2019) 003.

[52] P. B. Gilkey, The Geometry of Spherical Space Form Groups, Series in Pure Mathematics, Vol. 28 (World Scientific, Singapore, 2018).

[53] Z.-C. Gu and X.-G. Wen, Symmetry-protected topological orders for interacting fermions: Fermionic topological nonlinear $\sigma$ models and a special group supercohomology theory, Phys. Rev. B 90, 115141 (2014).

[54] D. Gaiotto and A. Kapustin, Spin TQFTs and fermionic phases of matter, Int. J. Mod. Phys. A 31, 1645044 (2016).

[55] N. Seiberg and E. Witten, Gapped boundary phases of topological insulators via weak coupling, Prog. Theor. Exp. Phys. 2016, 12C101 (2016).

[56] M. A. Metlitski, S-duality of $u(1)$ gauge theory with $\theta=\pi$ on non-orientable manifolds: Applications to topological insulators and superconductors, arXiv:1510.05663.

[57] Q.-R. Wang and Z.-C. Gu, Construction and Classification of Symmetry Protected Topological Phases in Interacting Fermion Systems, Phys. Rev. X 10, 031055 (2020).

[58] R. Thorngren, Anomalies and bosonization, arXiv:1810.04414.

[59] D. Delmastro, D. Gaiotto, and J. Gomis, Global anomalies on the Hilbert space, arXiv:2101.02218.

[60] B.-B. Mao and C. Wang, Mirror anomaly in fermionic topological orders, Phys. Rev. Res. 2, 023339 (2020).

[61] R. Kobayashi, Commuting projector models for $(3+1)$ dimensional topological superconductors via a string net of (1+1)-dimensional topological superconductors, Phys. Rev. B 102, 075135 (2020). 